Research article

\title{
A multicenter, double-blind, randomized, controlled phase III clinical trial of chicken type II collagen in rheumatoid arthritis
}

\author{
Wei Wei ${ }^{1}$, Ling-Ling Zhang ${ }^{1}$, Jian-Hua Xu${ }^{2}$, Feng Xiao ${ }^{1}$, Chun-De Bao ${ }^{3}$, Li-Oing Ni ${ }^{4}$, Xing-Fu Li ${ }^{5}$, \\ Yu-Qing Wu' ${ }^{6}$, Ling-Yun Sun ${ }^{7}$, Rong-Hua Zhang ${ }^{8}$, Bao-Liang Sun ${ }^{9}$, Sheng-Qian $X_{u^{2}}$, Shang Liu ${ }^{2}$, \\ Wei Zhang ${ }^{3}$, Jie Shen ${ }^{4}$, Hua-Xiang Liu ${ }^{5}$ and Ren-Cheng Wang ${ }^{9}$
}

\author{
${ }^{1}$ Institute of Clinical Pharmacology, Anhui Medical University, Key Laboratory of Anti-inflammatory and Immunopharmacology of Education Ministry, \\ 81 Meishan Road, Hefei 230032, PR China \\ ${ }^{2}$ Rheumatism and Immunity Department, The First Affiliated Hospital of Anhui Medical University, 218 Jixi Road, Hefei 230022, PR China \\ ${ }^{3}$ Rheumatism and Immunity Department, The Affiliated Shanghai Renji Hospital of Shanghai Jiao Tong University, 1630 Dongfang Road, Shanghai \\ 200127, PR China \\ ${ }^{4}$ Rheumatism and Immunity Department, Shanghai Guanghua Hospital, 540 Xinhua Road, Shanghai 200052, PR China \\ ${ }^{5}$ Rheumatism and Immunity Department, Qilu Hospital of Shandong University, 107 Wenhua Road, Jinan 250012, PR China \\ ${ }^{6}$ Rheumatism and Immunity Department, The Third Affiliated Hospital of Sun Yat-Sen University, 600 Tianhe Road, Guangzhou 510630, PR China \\ ${ }^{7}$ Rheumatism and Immunity Department, The Affiliated Drum Tower Hospital of Nanjing University Medical School, 321 Zhongshan Road, Nanjing \\ 210008, PR China \\ ${ }^{8}$ Rheumatism and Immunity Department, Southwest Hospital of Third Military Surgeon University, 30 Shapingba Gaotanyan Street, Chongqing \\ 400038, PR China \\ ${ }^{9}$ Rheumatism and Immunity Department, The Affiliated Hospital of Taishan Medical College, 706 Tanshan Street, Taian 271000, PR China
}

Corresponding author: Wei Wei, wwei@ahmu.edu.cn

Received: 10 Jul 2009 Revisions requested: 18 Aug 2009 Revisions received: 29 Sep 2009 Accepted: 1 Dec 2009 Published: 1 Dec 2009

Arthritis Research \& Therapy 2009, 11:R180 (doi:10.1186/ar2870)

This article is online at: http://arthritis-research.com/content/11/6/R180

(c) 2009 Wei et al.; licensee BioMed Central Ltd.

This is an open access article distributed under the terms of the Creative Commons Attribution License (http://creativecommons.org/licenses/by/2.0), which permits unrestricted use, distribution, and reproduction in any medium, provided the original work is properly cited.

\begin{abstract}
Introduction Chicken type II collagen (CCII) is a protein extracted from the cartilage of chicken breast and exhibits intriguing possibilities for the treatment of autoimmune diseases by inducing oral tolerance. A 24-week, double-blind, doubledummy, randomized, methotrexate (MTX)-controlled study was conducted to evaluate the efficacy and safety of CCII in the treatment of rheumatoid arthritis (RA).

Methods Five hundred three RA patients were included in the study. Patients received either $0.1 \mathrm{mg}$ daily of CCII $(n=326)$ or $10 \mathrm{mg}$ once a week of MTX $(n=177)$ for 24 weeks. Each patient was evaluated for pain, morning stiffness, tender joint count, swollen joint count, health assessment questionnaire (HAQ), assessments by investigator and patient, erythrocyte sedimentation rate (ESR), and C-reactive protein (CRP) by using the standard tools at baseline (week 0) and at weeks 12 and 24. Additionally, rheumatoid factor (RF) was evaluated at weeks 0 and 24. Measurement of a battery of biochemical parameters in serum, hematological parameters, and urine analysis was performed to evaluate the safety of CCII.
\end{abstract}

Results Four hundred fifty-four patients (94.43\%) completed the 24-week follow-up. In both groups, there were decreases in pain, morning stiffness, tender joint count, swollen joint count, $\mathrm{HAQ}$, and assessments by investigator and patient, and all differences were statistically significant. In the MTX group, ESR and CRP decreased. RF did not change in either group. At 24 weeks, $41.55 \%$ of patients in the CCIl group and $57.86 \%$ in the MTX group met the American College of Rheumatology 20\% improvement criteria (ACR-20) and $16.89 \%$ and $30.82 \%$, respectively, met the ACR 50\% improvement criteria (ACR-50). Both response rates for ACR-20 and ACR-50 in the CCll group were lower than those of the MTX group, and this difference was statistically significant $(P<0.05)$. The DAS28 (disease activity score using 28 joint counts) values of the two treatment groups were calculated, and there was a statistically significant difference between the two treatment groups $(P<0.05)$. Gastrointestinal complaints were common in both groups, but there were fewer and milder side effects in the CCll group than in the MTX group. The incidence of adverse events between the two groups was statistically significant $(P<0.05)$.

ACR: American College of Rheumatology; ACR-20: American College of Rheumatology 20\% improvement criteria; ACR-50: American College of Rheumatology 50\% improvement criteria; CCIl: chicken type II collagen; CIA: collagen-induced arthritis; Cll: type II collagen; CRP: C-reactive protein; DAS28: disease activity score using 28 joint counts; ESR: erythrocyte sedimentation rate; HAQ: health assessment questionnaire; IL: interleukin; ITT: intention-to-treat; MTX: methotrexate; NSAID: non-steroidal anti-inflammatory drug; RA: rheumatoid arthritis; RF: rheumatoid factor; TGF- $\beta$ : transforming growth factor-beta; Treg: regulatory T; VAS: visual analogue scale. 
Conclusions $\mathrm{CCll}$ is effective in the treatment of RA and is safe for human consumption. CCll exerts its beneficial effects by controlling inflammatory responses through inducing oral tolerance in RA patients.
Trials Registration Clinical trial registration number: ChiCTRTRC-00000093.

\section{Introduction}

Rheumatoid arthritis (RA) is a chronic inflammatory disease characterized by pain, swelling, and stiffness of multiple joints. It is also a highly disabling disease that limits mobility, hampers work, and reduces quality of life. Chronic inflammation commonly results in progressive joint destruction, deformity, and loss of function. Complex immune mechanisms contribute to the pathology of RA [1,2]. Current pharmacological strategies addressing mainly immune suppression and anti-inflammatory mechanisms have had limited success. Currently, most drugs for RA are steroids, non-steroidal anti-inflammatory drugs (NSAIDs), disease-modifying drugs, and biological agents. These therapies are associated with significant side effects with long administration, including anorexia, dyspepsy, suppression of the immune system non-specifically, and infections [3-5].

Recently, more and more oral tolerance mechanisms have been studied in the treatment of autoimmune diseases. Oral tolerance has posed intriguing possibilities for the treatment of autoimmune diseases, including RA. Oral tolerance is a state of systemic immune suppression to an antigen induced by oral feeding of the same antigen. Extensive research in this area over the past 10 years has led to the conclusion that two mechanisms are operative in the mediation of oral tolerance: active suppression and clonal anergy or deletion. A number of factors that determine which mechanisms of tolerance are operative have been identified: antigen dose, antigen form, and the timing of antigen administration $[6,7]$.

Oral administration of autoantigen has been shown to suppress a variety of autoimmune pathologies induced experimentally, including antigen-induced RA [8]. Modulating the immune response to the autoantigen by oral tolerance may be a safer and more effective treatment. A number of candidate autoantigens have been identified in RA [9]. Type II collagen (CII) is a major protein in articular cartilage and a potential autoantigen. Some RA patients demonstrate immunity against Cll, and autoantibodies to $\mathrm{Cll}$ have been detected in the sera of both pauciarticular-onset and systemic-onset RA patients [10]. These data support the view that autoimmunity to an antigen such as $\mathrm{Cll}$ in cartilage plays a major role in the pathogenesis of RA. In animal models, oral administration of Cll prevents and reduces the severity of autoimmune diseases [11]. Work from these animal models has recently been extended into human clinical trials of RA with differing degrees of success [12-14]. Hence, oral tolerance has been advocated as a treatment strategy for autoimmune diseases, including RA.
Investigators in our laboratory found that collagen-induced arthritis (CIA) could be established in Wistar rats, Kunming mice, and DBA/1 mice with chicken type II collagen (CCII) $[15,16]$. Feeding CCll to rats by oral administration decreased the arthritis index. Meanwhile, cartilage degeneration, synovium hyperplasia, and inflammatory cell infiltration in the knee joints of mice and rats with $\mathrm{ClA}$ were suppressed by $\mathrm{CCll}$ $[17,18]$. These experiments in rodents have provided the basis for human clinical trials. In a randomized, double-blind, multicenter, and controlled phase II clinical trial involving 236 patients with severe active RA, a decrease in the number of swollen joints and tender joints occurred in subjects fed CCll for 6 months. Meanwhile, CCll could reduce pain, morning stiffness, health assessment questionnaire (HAQ), and assessments by investigator and patient, and the incidence of adverse events of CCll was lower than that of methotrexate (MTX) [19]. These results demonstrate clinical efficacy of an oral tolerance approach for RA. To evaluate the efficacy and safety of CCll in RA patients further, we treated two groups of RA patients with oral CCII or MTX in a randomized, doubleblind, multicenter, and controlled phase III clinical trial.

\section{Materials and methods Recruitment of patients}

This trial was performed at eight centers from October 2004 to December 2005 (clinical trial registration number: ChiCTRTRC-00000093). The study protocol was evaluated and approved by their respective investigational and ethics committees. Five hundred three intention-to-treat (ITT) population RA patients (18 to 65 years old) who met revised American College of Rheumatology (ACR) criteria for the diagnosis of RA were entered into the study after giving their written informed consent [20]. There are no patients in the phase II study who were enrolled in this phase III trial. Table 1 defines the study population. Admission criteria also included patients of either gender with RA with a duration of 6 to 24 months. Active RA was defined as the presence of at least three of the following criteria: six or more painful or tender joints, three or more swollen joints, morning stiffness for at least 45 minutes (on average during the week prior to entry), and an erythrocyte sedimentation rate (ESR) of at least $28 \mathrm{~mm}$. Second-line agents were discontinued at least 4 weeks prior to entry. Continuous doses of NSAIDs were permitted. Patients to whom one of following applied were excluded: dysfunction of liver; severe cardiovascular, urinary, hematopoietic, or endocrine system disease; immunodeficiency; uncontrolled infection or active gastrointestinal tract disease; recent vaccination; gravida; women in lactation period or those recently intending to 
Table 1

\begin{tabular}{|c|c|c|c|}
\hline Variables & CCII $(n=296)$ & MTX $(n=158)$ & $P$ value \\
\hline Gender, male/female & $55 / 241$ & $30 / 128$ & 0.983 \\
\hline Age, years & $47.11 \pm 10.55$ & $47.06 \pm 11.14$ & 0.967 \\
\hline Duration of rheumatoid arthritis, years & $1.63 \pm 0.75$ & $1.72 \pm 0.52$ & 0.949 \\
\hline Body temperature, ${ }^{\circ} \mathrm{C}$ & $36.66 \pm 0.38$ & $36.65 \pm 0.39$ & 0.892 \\
\hline Pain (VASa) & $6.02 \pm 1.43$ & $5.91 \pm 1.76$ & 0.528 \\
\hline Morning stiffness, minutes & $99.26 \pm 25.14$ & $104.89 \pm 26.42$ & 0.277 \\
\hline Tender joint count & $13.34 \pm 6.43$ & $14.09 \pm 6.82$ & 0.233 \\
\hline Swollen joint count & $10.38 \pm 6.63$ & $10.57 \pm 7.25$ & 0.773 \\
\hline $\mathrm{HAQ}^{\mathrm{b}}$ & $0.82 \pm 0.56$ & $0.86 \pm 0.55$ & 0.454 \\
\hline Physician's assessment (VAS) & $5.83 \pm 1.54$ & $5.78 \pm 1.26$ & 0.892 \\
\hline Patient's assessment (VAS) & $6.01 \pm 1.51$ & $6.06 \pm 1.71$ & 0.731 \\
\hline $\mathrm{ESR}^{\mathrm{c}}, \mathrm{mm} /$ hour & $38.18 \pm 27.58$ & $42.51 \pm 29.37$ & 0.118 \\
\hline C-reactive protein, $\mathrm{mg} / \mathrm{L}$ & $17.52 \pm 8.27$ & $23.24 \pm 9.81$ & 0.306 \\
\hline Rheumatoid factor, $\mathrm{U} / \mathrm{mL}$ & $203.65 \pm 61.95$ & $198.99 \pm 21.31$ & 0.115 \\
\hline
\end{tabular}

The same variables were compared between the chicken type II collagen (CCII) group and the methotrexate (MTX) group. Fisher exact test was used for categorical variables, and $t$ analysis of variance was used for continuous variables. ${ }^{\circ} E S R$, erythrocyte sedimentation rate; bHAQ, health assessment questionnaire; MTX, methotrexate; aVAS, visual analogue scale.

become pregnant; hypersensitivity to Cll; treatment with any other disease-modifying anti-rheumatic drug within 30 days before enrolment; history of alcohol abuse; history of hyperglycemia or motor coordination disorder; or participation in other clinical trials within 3 months before enrolment.

\section{Study design}

The study was a two-to-one, eight-center, 24-week follow-up, double-blind, double-dummy, randomized, and MTX-controlled trial comparing efficacy and safety of CCII and MTX in the treatment of RA. Patients were randomly assigned to a CCII ( $\mathrm{n}$ $=326)$ or MTX $(n=177)$ group that received either CCII $(0.1$ mg daily) or MTX (10 mg once a week). Patients and investigators were blinded to the treatment regimens throughout the study. Efficacy variables were assessed at 0,12 , and 24 weeks after administration of drug. Patients were allowed to remain on diclofenac sodium ( $50 \mathrm{mg}$ daily), an NSAID. The diclofenac sodium dosage was not changed during the study. CCll capsules (\#040328; Shanghai Materia Medica Bioengineering Institute, Shanghai, China), CCll dummy capsules, MTX tablets (\#031201; Shanghai Xin Yi Pharmaceutical Factory, Shanghai, China), and MTX dummy tablets were obtained from Shanghai Materia Medica Bioengineering Institute. Patients were instructed to take oral CCll capsules or dummy capsules with $200 \mathrm{~mL}$ of cold water 30 minutes prior to eating breakfast every morning.
Flow sheet of production of chicken type II collagen

$\mathrm{CCll}$ is a protein extracted from the cartilage of chicken breast. Its molecular weight is 115 to approximately $135 \mathrm{kDa}$ by SDSPAGE electrophoresis method. In this study, the CCll capsule that patients received consisted of $\mathrm{CCll}$ and an adjuvant such as mannitol and glidantin. Figure 1 shows the flow sheet of production of CCII.

\section{Clinical assessments}

Clinical assessments of efficacy were made at baseline and repeated 12 and 24 weeks later. Efficacy variables included $[21,22]$ pain, and pain intensity was assessed by visual analogue scale (VAS) of 0 (no pain) to 10 (severe pain). Patients were questioned about the duration of morning stiffness experienced on the day before each study visit. Joint counts for tenderness and swelling were the sum of the number of affected joints. Physician and patient global assessments were rated according to VAS of 0 (very good) to 10 (very poor). Functional status was assessed at baseline and at 12 and 24 weeks using $\mathrm{HAO}$. ESR and C-reactive protein (CRP) values were obtained at baseline and at 12 and 24 weeks. Rheumatoid factor (RF) positivity was determined at the screening visit and at 24 weeks.

The primary efficacy variable was the ACR preliminary definition of improvement in RA [23]. To reach improvement according to the ACR definition, a patient with RA must improve by at least $20 \%$ in tender and swollen joint count and by at least $20 \%$ in three of the five other measures: patient global assess- 
Figure 1

Cartilage of chicken breast is gained by washout and cleaning $\downarrow$

Cartilage is placed in guanidine hydrochloride-Tris

$$
\downarrow
$$

Proteoglycans are removed

$\downarrow$

Cartilage is degradated in acetic acid $(0.5 \mathrm{~mol} / \mathrm{L})$

$\downarrow$

Solution of degradation containing CC II

$\downarrow$

CC II is isolated by salting-out (sodium chloride $0.7 \mathrm{~mol} / \mathrm{L}$ ) three times. $\downarrow$

Precipitation of CC II

$\downarrow$

Precipitation of CC II is dissolved and dialyzed

$\downarrow$

Solution containing CC II is lyophilized

$$
\text { Crude CC II }
$$

$\downarrow$

Mannitol is added

$\downarrow$

The product is lyophilized

$\downarrow$

Raw material of capsule is added

$\downarrow$

The capsule of CC II is made.

The flow sheet of production of chicken type II collagen (CCII). CCII is a protein extracted from the cartilage of chicken breast. Its molecular weight is 115 to approximately $135 \mathrm{kDa}$ by SDS-PAGE electrophoresis method. In this study, the CCll capsule that patients received consisted of CCII and an adjuvant such as mannitol and glidantin.

ment, physician global assessment, $\mathrm{HAQ}$, acute-phase reactant, and patient pain assessment. In addition to the evaluation of $20 \%$ improvement (ACR-20), we determined RA improvement based on more substantial changes in RA core set measures, such as requiring at least 50\% improvement (ACR-50) reported as secondary efficacy measures. The disease activity score using 28 joint counts (DAS28) was evaluated [24]. Clinical parameters also included body weight, blood pressure, and heart rate. To standardize the evaluation of clinical variables, all investigators prior to study entry performed clinical evaluation of one patient with active RA.

\section{Adverse events}

At each visit, the patient was asked whether side effects were noticed during the interim. Side effects such as gastrointestinal complaints, vomiting, anorexia, headache, dizziness, insomnia, tetter, and mouth ulcers were known to occur frequently in treatment with CCll or MTX. Moreover, at entry and at 12 and 24 weeks, the following laboratory variables were assessed to monitor safety: complete blood cell count, serum levels of liver enzymes, creatinine, uric acid, and urinalysis.

\section{Statistical analysis}

Safety assessments were performed on all patients who consumed any masked study medication. Efficacy analyses were performed on the ITT population as well as on the population of patients who completed the 24-week study. Efficacy analysis of outcome variables was based on mean changes from baseline to endpoint in the ITT population. The data in Tables 1 and 2 and Figure 2 are expressed as mean \pm standard deviation. The statistical software product used for these analyses was SAS, version 8.1 (SAS Institute Inc., Cary, NC, USA). All laboratory variables were subjected to descriptive statistics and compared by means of the Wilcoxon signed rank test. The randomization code was exposed only after the database was locked. Chi-square with Fisher exact test was used for categorical variables, and $t$ analysis of variance was used for continuous variables. Significance level was established at 0.05 .

\section{Results \\ Baseline characteristics}

Of 503 randomly assigned patients (326 in the CCIl group and 177 in the MTX group), 49 patients withdrew early. Thirty $(9.20 \%)$ patients withdrew in the CCll group, and 19 $(10.73 \%)$ patients withdrew in the MTX group. There were various reasons for early withdrawal, such as adverse events, non-compliance, lack of response, and loss at follow-up. Three patients $(0.92 \%)$ in the CCll group reported side effects, and five patients $(2.82 \%)$ in the MTX group reported side effects. In the CCll group, three patients $(0.92 \%)$ withdrew due to lack of compliance, and four patients (2.25\%) withdrew because of non-compliance in the MTX group. Twelve (3.68\%) and four $(2.25 \%)$ patients in the CCll group and in the MTX group, respectively, withdrew because of lack of efficacy. Twelve and six patients in the CCIl group and in the MTX group, respectively, were lost to follow-up. Four hundred fifty-four patients (296 in the CCll group and 158 in the MTX group) completed 24 weeks of therapy. There were no statistically significant differences between the two groups in terms of adverse events, non-compliance, lack of response, and loss at follow-up. At study entry, the two groups were well balanced with regard to demographic characteristics and disease parameters, and there were no statistically significant differences between the two groups in terms of gender, age, disease duration, body temperature, pain, morning stiffness, tender joint count, swollen joint count, HAQ, physician's assessment, patient's assessment, ESR, CRP, and RF (Table 1). Also, there were no important differences among the eight centers.

\section{Efficacy}

In both groups, there were decreases in pain, morning stiffness, tender joint count, swollen joint count, $\mathrm{HAQ}$, and assessments of efficacy by both investigator and patient. Within-group differences (study entry versus 12 and 24 weeks) were statistically significant for the above clinical disease parameters (Table 2). At 12 weeks, there were statistically significant differences in morning stiffness, swollen joint count, HAQ, physician's assessment, and patient's assessment between the CCll group and the MTX group; there were statistically significant differences in pain, $\mathrm{HAQ}$, and patient's 
Table 2

\begin{tabular}{|c|c|c|c|c|c|}
\hline Outcome variables & CClI & $P$ value ${ }^{a}$ & MTX & $P$ value ${ }^{a}$ & $P$ value $^{\mathrm{b}}$ \\
\hline \multicolumn{6}{|l|}{ Pain (VAS) } \\
\hline Entry & $6.02 \pm 1.43$ & & $5.91 \pm 1.76$ & & $>0.05$ \\
\hline 12 weeks & $4.59 \pm 2.22$ & $<0.01$ & $4.09 \pm 1.99$ & $<0.01$ & $>0.05$ \\
\hline 24 weeks & $3.58 \pm 2.55$ & $<0.01$ & $3.38 \pm 2.35$ & $<0.01$ & $<0.05$ \\
\hline \multicolumn{6}{|c|}{ Morning stiffness, minutes } \\
\hline Entry & $99.26 \pm 25.14$ & & $104.89 \pm 26.42$ & & $>0.05$ \\
\hline 12 weeks & $62.66 \pm 25.06$ & $<0.01$ & $45.83 \pm 21.28$ & $<0.01$ & $<0.01$ \\
\hline 24 weeks & $36.12 \pm 17.21$ & $<0.01$ & $33.98 \pm 12.59$ & $<0.01$ & $>0.05$ \\
\hline \multicolumn{6}{|l|}{ Tender joint count } \\
\hline Entry & $13.34 \pm 6.43$ & & $14.09 \pm 6.82$ & & $>0.05$ \\
\hline 12 weeks & $9.14 \pm 6.67$ & $<0.01$ & $8.78 \pm 6.01$ & $<0.01$ & $>0.05$ \\
\hline 24 weeks & $6.34 \pm 4.81$ & $<0.01$ & $7.22 \pm 6.91$ & $<0.01$ & $>0.05$ \\
\hline \multicolumn{6}{|l|}{ Swollen joint count } \\
\hline Entry & $10.38 \pm 6.63$ & & $10.57 \pm 7.25$ & & $>0.05$ \\
\hline 12 weeks & $6.89 \pm 5.46$ & $<0.01$ & $5.63 \pm 4.97$ & $<0.01$ & $<0.01$ \\
\hline 24 weeks & $4.26 \pm 2.03$ & $<0.01$ & $4.38 \pm 2.94$ & $<0.01$ & $>0.05$ \\
\hline \multicolumn{6}{|l|}{$\mathrm{HAQ}$} \\
\hline Entry & $0.82 \pm 0.56$ & & $0.86 \pm 0.55$ & & $>0.05$ \\
\hline 12 weeks & $0.65 \pm 0.41$ & $<0.01$ & $0.51 \pm 0.42$ & $<0.01$ & $<0.01$ \\
\hline 24 weeks & $0.43 \pm 0.27$ & $<0.01$ & $0.44 \pm 0.21$ & $<0.01$ & $<0.05$ \\
\hline \multicolumn{6}{|c|}{ Physician's assessment (VAS) } \\
\hline Entry & $5.83 \pm 1.54$ & & $5.78 \pm 1.26$ & & $>0.05$ \\
\hline 12 weeks & $4.68 \pm 2.09$ & $<0.01$ & $4.03 \pm 2.06$ & $<0.01$ & $<0.01$ \\
\hline 24 weeks & $3.81 \pm 1.52$ & $<0.01$ & $3.53 \pm 1.64$ & $<0.01$ & $>0.05$ \\
\hline \multicolumn{6}{|c|}{ Patient's assessment (VAS) } \\
\hline Entry & $6.01 \pm 1.51$ & & $6.06 \pm 1.71$ & & $>0.05$ \\
\hline 12 weeks & $4.86 \pm 2.01$ & $<0.01$ & $4.31 \pm 2.06$ & $<0.01$ & $<0.01$ \\
\hline 24 weeks & $3.92 \pm 2.45$ & $<0.01$ & $3.71 \pm 2.04$ & $<0.01$ & $<0.05$ \\
\hline \multicolumn{6}{|l|}{$\mathrm{ESR}^{\mathrm{c}}, \mathrm{mm} / \mathrm{hour}$} \\
\hline Entry & $38.18 \pm 17.58$ & & $42.51 \pm 19.37$ & & $>0.05$ \\
\hline 12 weeks & $38.03 \pm 14.17$ & $>0.05$ & $35.84 \pm 13.24$ & $<0.01$ & $<0.01$ \\
\hline 24 weeks & $37.53 \pm 10.22$ & $>0.05$ & $34.21 \pm 15.25$ & $<0.01$ & $<0.01$ \\
\hline \multicolumn{6}{|c|}{ C-reactive protein, $\mathrm{mg} / \mathrm{L}$} \\
\hline Entry & $17.52 \pm 8.27$ & & $23.24 \pm 9.81$ & & $>0.05$ \\
\hline 12 weeks & $17.27 \pm 15.14$ & $>0.05$ & $16.01 \pm 13.41$ & $<0.01$ & $<0.05$ \\
\hline 24 weeks & $15.56 \pm 12.38$ & $>0.05$ & $15.08 \pm 12.25$ & $<0.01$ & $<0.05$ \\
\hline \multicolumn{6}{|c|}{ Rheumatoid factor, $\mathrm{U} / \mathrm{mL}$} \\
\hline Entry & $203.65 \pm 61.95$ & & $198.99 \pm 21.31$ & & $>0.05$ \\
\hline 24 weeks & $150.21 \pm 16.18$ & $>0.05$ & $123.35 \pm 11.32$ & $>0.05$ & $>0.05$ \\
\hline
\end{tabular}

In both groups, there were decreases in pain, morning stiffness, tender joint count, swollen joint count, health assessment questionnaire (HAO), and global assessment of efficacy by investigator and patient. Within-group differences (study entry versus 12 and 24 weeks) were statistically significant. In the methotrexate (MTX) group, erythrocyte sedimentation rate (ESR) and C-reactive protein decreased, but changes in the two variables in the chicken type II collagen (CCII) group were not significant. Rheumatoid factor was not significantly affected by either drug therapy. VAS, visual analogue scale. $P$ valuea, entry versus 12 or 24 weeks in CCIl group or MTX group; $P$ value ${ }^{b}$, CCIl group versus MTX group;“CESR, erythrocyte sedimentation rate. 


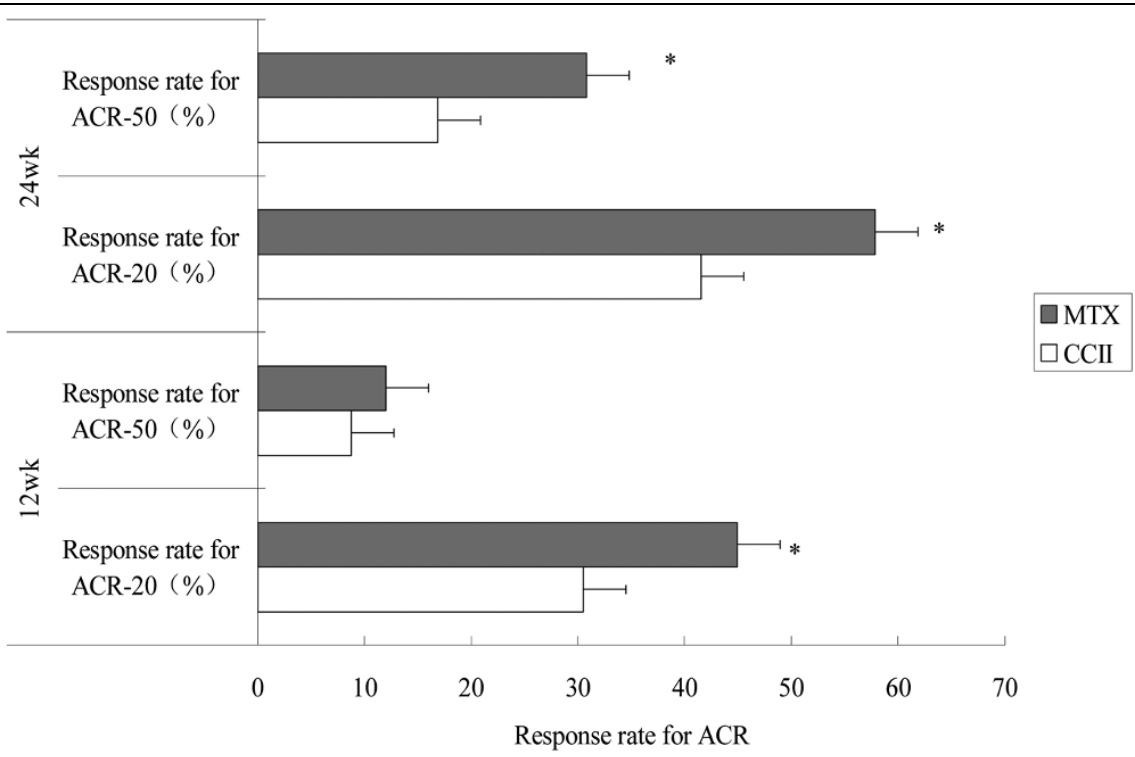

Comparison of the effect of American College of Rheumatology 20\% improvement criteria (ACR-20) and ACR 50\% improvement criteria (ACR-50) between two groups at 12 and 24 weeks. Response rates for ACR-20 and ACR-50 were assessed at 12 and 24 weeks. With an intention-to-treat analysis, response rates for ACR-20 were $30.51 \%$ in the chicken type II collagen (CCII) group and $44.94 \%$ in the methotrexate (MTX) group at 12 weeks. Response rates for ACR-50 were $8.81 \%$ and $15.03 \%$, respectively. Response rates for ACR-20 were $41.55 \%$ in the CCIl group and $57.86 \%$ in the MTX group at 24 weeks. Response rates for ACR-50 were $16.89 \%$ and $30.82 \%$, respectively. Both response rates for ACR-20 and ACR-50 of the CCll group were lower than those of the MTX group. These changes were different to a statistically significant degree between the two treatment groups. ${ }^{*} P<0.05$ versus $\mathrm{CCll}$ group.

assessment at 24 weeks between the two groups. In the MTX group, ESR and CRP decreased at 12 and 24 weeks, but changes in the two variables in the CCll group were not significant, and there was a statistically significant difference between the two groups. RF was not significantly affected by either drug therapy (Table 2).

\section{American College of Rheumatology response criteria}

Response rates for ACR- 20 and ACR-50 were assessed at 12 and 24 weeks. With an ITT analysis (Figure 2), response rates for ACR-20 were $30.51 \%$ in the CCll group and $44.94 \%$ in the MTX group at 12 weeks. Response rates for ACR-50 were $8.81 \%$ and $15.03 \%$, respectively. Response rates for ACR-20 were $41.55 \%$ in the CCll group and $57.86 \%$ in the MTX group at 24 weeks. Response rates for ACR-50 were $16.89 \%$ and $30.82 \%$, respectively. Both response rates for ACR-20 and ACR-50 of the CCll group were lower than those of the MTX group. These changes were different to a statistically significant degree between the two treatment groups $(P$ $<0.05)$.

\section{Disease activity score using 28 joint counts}

The DAS28 values of the two treatment groups were calculated (Table 3 ), and the results showed that the numbers of relief patients $(\leq 2.6)$, low-activity patients (2.6 to approximately 3.2), mid-activity patients (3.2 to approximately 5.1 ), and high-activity patients $(>5.1)$ were $36,26,138$, and 96, respectively, in the CCII group at 24 weeks. In the MTX group, the numbers of relief patients, low-activity patients, mid-activity patients, and high-activity patients were 20, 19, 88, and 31, respectively. There was a statistically significant difference between the two treatment groups $(P<0.05)$.

Table 3

Comparison of DAS28 between the chicken type II collagen group and the methotrexate group

\begin{tabular}{|c|c|c|c|c|c|c|}
\hline Group & $\begin{array}{l}\text { Relief } \\
(\leq 2.6)\end{array}$ & $\begin{array}{c}\text { Low activity } \\
(2.6 \sim 3.2)\end{array}$ & $\begin{array}{l}\text { Mid activity } \\
(3.2 \sim 5.1)\end{array}$ & $\begin{array}{l}\text { High activity } \\
(>5.1)\end{array}$ & Chi-square & $P$ value $^{\mathrm{a}}$ \\
\hline $\mathrm{CCll}(n=296)$ & 36 & 26 & 138 & 96 & 5.476 & 0.019 \\
\hline $\operatorname{MTX}(n=158)$ & 20 & 19 & 88 & 31 & & \\
\hline
\end{tabular}

aShowed rank sum test. CCII, chicken type II collagen; DAS28, disease activity score using 28 joint counts; MTX, methotrexate. 


\section{Adverse events}

All trials reported data about drug-related adverse outcomes in the ITT population. The majority of adverse outcomes were mild to moderate disturbances of the gastrointestinal tract. Gastrointestinal complaints were the most common adverse events. Other adverse events included vomiting, anorexia, headache, dizziness, insomnia, tetter, and mouth ulcers. These adverse events were mild and did not interfere with the continuation of treatment drugs (Table 4).

The analysis of adverse events was carried out on all 503 randomly assigned patients, and the adverse events of all patients were generally well tolerated. Adverse events were common in both groups. During the treatment period, the CCII group reported $18(5.52 \%)$ adverse events whereas the MTX group reported $15(8.47 \%)$ at 12 weeks. There were $18(5.52 \%)$ adverse events in the CCll group and 17 (9.60\%) adverse events in the MTX group at 24 weeks. There were fewer and milder side effects in the CCll group than in the MTX group (Table 5). The incidence of adverse events between the CCII group and MTX group was statistically significant $(P<0.05)$.

\section{Laboratory variables}

In the MTX group, there was a significant increase from baseline in transaminase and a decrease in white blood cell count. There were decreases in hemoglobin, platelet count, and neutrophil count in both groups, but the differences were insignificant (Table 4). Other laboratory variables were not significantly affected in the two groups.

\section{Discussion}

Oral tolerance has been applied to prevent and treat autoimmune disease in several animal models, including arthritis. Cll is the most abundant structural protein of human cartilage. The cartilage within the joint caused mainly damage of autoimmunity in patients with RA. Cll autoimmunity may be a secondary phenomenon induced following inflammation in the joints and may play a role in the persistence of the disease rather than in actual induction of arthritis [25,26]. In either case, downregulation of the immune response to $\mathrm{Cll}$ may help prevent the resulting destructive arthritis. Oral administration of $\mathrm{Cll}$ is an established procedure for inducing peripheral immune tolerance, which suppresses autoimmune responses in RA [27-
29]. Bovine or chicken Cll has been administered orally to RA patients, resulting in some clinical improvement. However, the precise mechanisms of oral tolerance are not fully known. Animal studies have revealed that the mechanisms of induction of oral tolerance include clonal deletion, suppression of the proinflammatory Th1 cells, and the induction of regulatory T (Treg) cells. Treg cells from Peyer's patches in the gut-associated lymphoid tissue and transforming growth factor-beta (TGF- $\beta$ ) are reported to mediate the induction of active suppression $[8,30]$. Pro-inflammatory cytokines such as interleukin (IL)-1 and tumor necrosis factor-alpha downregulate and suppressive cytokines such as TGF- $\beta$ and IL-4 upregulate in oral tolerance. Treg cells, defined as a persistently CD25-expressing subset of $\mathrm{CD}^{+}$cells, may produce anti-inflammatory cytokines such as IL-10 and TGF- $\beta$ and are likely to be agents of bystander suppression [31]. In a basic study, investigators in our laboratory found that CCll plays an important role in regulating the immune balance of Th1/Th2 and Th17/Treg in rats with CIA. CCll decreases the overproduction of pro-inflammatory mediator (IL-2, IL-17) and increases the hypoproduction of anti-inflammatory mediator (IL-4, TGF- $\beta$ ). These results may support a mechanism of oral tolerance [32].

A key feature that may affect the induction of Treg cells and other suppressive mechanisms is the dose of antigen administered. A low dose of antigen stimulates the development of Treg cells, leading to an active immune suppression. The active mechanism appears to be a cytokine-mediated immune deviation with a predominant Th2 and Th3 response (TGF- $\beta$ ). In contrast, high-dose oral antigens lead to clonal deletion and anergy $[33,34]$. The active suppression of low-dose oral tolerance can also suppress an unrelated immune response (bystander suppression), paving the way for therapy of autoimmune diseases [35]. The results from human clinical trials suggest that a daily dose of significantly less than $1 \mathrm{mg}$ is optimal. Similarly, data from CIA studies reveal an optimal dose above and below which there is little or no immune suppression. Indeed, the incorrect dose can prime the immune response and aggravate disease. The timing and frequency of administration are also vital to the level of immune tolerance induced and the control of the pathological process [36].

Table 4

Comparison of adverse events between the chicken type II collagen group and the methotrexate group at 12 and 24 weeks

\begin{tabular}{llccc}
\hline Weeks & Groups & Adverse events & Rate of adverse events & Chi-square $^{P} \boldsymbol{P}_{\text {value }}{ }^{\mathbf{a}}$ \\
\hline 12 & CCII $(\mathrm{n}=326)$ & 18 & $5.52 \%$ & 1.974 \\
& MTX $(\mathrm{n}=177)$ & 15 & $8.47 \%$ & \\
24 & CCII $(n=326)$ & 18 & $5.52 \%$ & 2.001 \\
& MTX $(n=177)$ & 17 & $9.60 \%$ & 0.036
\end{tabular}

aShowed chi-square test. CCII, chicken type II collagen; MTX, methotrexate. 
Table 5

\begin{tabular}{|c|c|c|}
\hline Adverse events & $\operatorname{CCII}(n=326)$ & $\operatorname{MTX}(n=177)$ \\
\hline Nausea, vomiting & 6 & 13 \\
\hline Abdominal pain, epigastric discomfort & 17 & 11 \\
\hline Diarrhea & 0 & 2 \\
\hline Liver dysfunction & 6 & 10 \\
\hline Headache, dizziness & 3 & 5 \\
\hline Itching, rash & 5 & 1 \\
\hline Blood in urine & 2 & 3 \\
\hline Constipation & 1 & 0 \\
\hline Edema & 2 & 1 \\
\hline Alopecia & 0 & 1 \\
\hline Decrease in white blood cell count & 1 & 5 \\
\hline Decrease in platelet count & 0 & 1 \\
\hline Sum of events & 43 & 53 \\
\hline
\end{tabular}

CCIl, chicken type II collagen; MTX, methotrexate.

In a multicenter study, Barnett and colleagues [14] treated 90 RA patients with CCll for 12 weeks. Patients taking the lowest dose of Cll $(20 \mu \mathrm{g})$ had a 'significant' improvement in response rate compared with placebo-treated control patients $(P<0.035)$. Corrigall and Panayi [37] also reported a significant improvement in a group of RA patients treated with lyophilized Cll. None of the patients in either study had significant side effects attributable to treatment with collagen. Similarly, Barnett and colleagues [13] previously reported that 8 of 10 juvenile RA patients had decreased numbers of swollen and tender joints after 3 months of treatment with CCII. Sieper and colleagues [38] administered bovine Cll orally to patients with early RA for 12 weeks using doses of either $1 \mathrm{mg} /$ day or $10 \mathrm{mg} /$ day. More patients in the Cll-treated groups met the ACR-20 and ACR-50 improvement criteria than did patients in the placebo group [23].

On the basis of the above, CCll was developed as a novel drug of immunologic tolerance. The present study was undertaken to further evaluate whether oral administration of $\mathrm{CCll}$ is safe and effective in patients with RA. In this phase III trial, CCII at $0.1 \mathrm{mg}$ daily and MTX at $10 \mathrm{mg}$ once weekly effectively alleviated signs and symptoms of active RA. However, the efficacy of CCll did not exceed that of MTX. The incidence of treatment-related adverse events was significantly different between the CCII group and the MTX group. According to the former study, CCll led to few adverse events in patients with RA [27]. In this study, treatment was carried out in combination with diclofenac sodium, an NSAID, which can frequently cause gastrointestinal complaints. The use of diclofenac sodium can relieve pain. Nevertheless, its mechanism of action and toxicity can overlap with those of the trial drugs. The effi- cacy of CCll will be affected since diclofenac sodium can damage the alimentary system [39].

Above all, treatment of autoimmune diseases by induction of oral tolerance is attractive because of the few side effects and easy clinical implementation of this approach. The MTX-controlled, multicenter, 24-week trial in RA patients confirms that treatment with oral Cll leads to improvement in arthritis and no significant side effects. These results are encouraging and imply that RA can be effectively treated with oral Cll and partly supported the mechanism of oral tolerance. These data will provide a basis for more effective application of oral tolerance induction in RA patients. However, to clarify further the potential role and effectiveness of $\mathrm{CCll}$ as a toleragen in RA, ongoing studies and future work should clarify the autoimmune response to collagen in the pathogenesis of RA, and long-term observations in large numbers of patients need to confirm the efficacy of CCll and determine the optimal doses of orally administered CCll and which patients with autoimmune diseases will profit most from it.

\section{Conclusions}

In summary, the present study provides evidence in support of the potential efficacy and safety of CCll in patients with RA. CCll significantly improved joint function and exhibited better therapeutic efficacy and is safe for human consumption, even in the long term. This study provides important information about the efficacy and safety of CCll in the treatment of RA, and this information may be useful in promoting $\mathrm{CCll}$ as a promising alternative therapeutic strategy that may be used as a nutritional supplement against RA. 


\section{Competing interests}

The authors declare that they have no competing interests.

\section{Authors' contributions}

WW contributed to the design of the project, served as the study coordinator, and helped to review the manuscript. LLZ contributed to the design of the project and was primarily responsible for writing the manuscript. JHX, CDB, LQN, XFL, YQW, LYS, RHZ, and BLS contributed to patient recruitment and management and to data collection. SQX, SL, WZ, JS, $\mathrm{HXL}$, and RCW worked with patients to obtain informed consent, conducted clinical evaluations, took samples, and evaluated the therapeutic response to CCII. FX contributed to clinical data analysis. All authors read and approved the final manuscript.

\section{Acknowledgements}

Gui-Jun Fei participated in the collection of data. This study was funded by Shanghai Materia Medica Bioengineering Institute and Shanghai Baolong Pharmacy Limited Company (Shanghai, China).

\section{References}

1. Turner DE, Helliwell PS, Woodburn J: Methodological considerations for a randomised controlled trial of podiatry care in rheumatoid arthritis: lessons from an exploratory trial. BMC Musculoskelet Disord 2007, 8:109-119.

2. Chung KC, Burns PB, Wilgis EF, Burke FD, Regan M, Kim HM, Fox DA: A multicenter clinical trial in rheumatoid arthritis comparing silicone metacarpophalangeal joint arthroplasty with medical treatment. J Hand Surg Am 2009, 34:815-823.

3. Grijalva CG, Chung CP, Arbogast PG, Stein CM, Mitchel EF Jr, Griffin MR: Assessment of adherence to and persistence on disease-modifying antirheumatic drugs (DMARDs) in patients with rheumatoid arthritis. Med Care 2007, 45:S66-76.

4. Boyer JF, Cantagrel A, Constantin A: Impact of traditional therapies and biologics on cardiovascular diseases in rheumatoid arthritis. Curr Vasc Pharmaco/ 2008, 6:218-227.

5. Edwards CJ, Cooper C, Fisher D, Field M, van Staa TP, Arden NK: The importance of the disease process and disease-modifying antirheumatic drug treatment in the development of septic arthritis in patients with rheumatoid arthritis. Arthritis Rheum 2007, 57:1151-1157.

6. Maier E, Reipert BM, Novy-Weiland T, Auer W, Baumgartner B, Muchitsch EM: Induction of immune tolerance by oral IVIG. Int Immunopharmacol 2007, 7:351-359.

7. Tsuji NM, Kosaka A: Oral tolerance: intestinal homeostasis and antigen-specific regulatory T cells. Trends Immunol 2008, 29:532-540.

8. Bagchi D, Misner B, Bagchi M, Kothari SC, Downs BW, Fafard $\mathrm{RD}$, Preuss HG: Effects of orally administered undenatured type II collagen against arthritic inflammatory diseases: a mechanistic exploration. Int J Clin Pharmacol Res 2002, 22:101-110.

9. Myers LK, Tang B, Rosioniec EF, Stuart JM, Kang AH: An altered peptide ligand of type II collagen suppresses autoimmune arthritis. Crit Rev Immuno/ 2007, 27:345-356.

10. Rowley MJ, Nandakumar KS, Holmdahl R: The role of collagen antibodies in mediating arthritis. Mod Rheumatol 2008, 18:429-441.

11. Ding CH, Li Q, Xiong ZY, Zhou AW, Jones G, Xu SY: Oral administration of type II collagen suppresses pro-inflammatory mediator production by synoviocytes in rats with adjuvant arthritis. Clin Exp Immunol 2003, 132:416-423.

12. Trentham DE: Evidence that type II collagen feeding can induce a durable therapeutic response in some patients with rheumatoid arthritis. Ann N Y Acad Sci 1996, 778:306-314.

13. Barnett ML, Combitchi D, Trentham DE: A pilot trial of oral type II collagen in the treatment of juvenile rheumatoid arthritis. Arthritis Rheum 1996, 39:623-628.
14. Barnett ML, Kremer JM, Trentham DE: Treatment of rheumatoid arthritis with oral type II collagen. Arthritis Rheum 1998, 41:290-297.

15. Chen $Q$, Wei W: Effects and mechanisms of glucosides of chaenomeles speciosa on collagen-induced arthritis in rats. Int Immunopharmacol 2003, 3:593-608.

16. Zhang LL, Wei W: Therapeutic effects of glucosides of cheanomeles speciosa on collagen-induced arthritis in mice. Acta Pharmacol Sin 2004, 25:1495-1501.

17. Zheng $Y Q$, Wei W, Shen $Y X$, Dai M, Liu LH: Oral and nasal administration of chicken type II collagen suppresses adjuvant arthritis in rats with intestinal lesions induced by meloxicam. World J Gastroenterol 2004, 10:3165-3170.

18. Ju JH, Cho ML, Jhun JY, Park MJ, Oh HJ, Min SY, Cho YG, Hwang SY, Kwok SK, Seo SH, Yoon CH, Park SH, Kim HY: Oral administration of type-II collagen suppresses IL-17-associated RANKL expression of CD4+ $\mathrm{T}$ cells in collagen-induced arthritis. Immunol Lett 2008, 117:16-25.

19. Zhang LL, Wei W, Xiao F, Xu JH, Bao CD, Ni LQ, Li XF: A randomized, double-blind, multicenter, controlled clinical trial of chicken type II collagen in rheumatoid arthritis. Arthritis Rheum 2008, 59:905-910.

20. Thompson DJ, Barron KS, Whitcup SM, Robinson MR: The safety and efficacy of chicken type II collagen on uveitis associated with juvenile rheumatoid arthritis. Ocul Immunol Inflamm 2002, 10:83-91.

21. Vliet Vlieland TP, Zwinderman AH, Breedveld FC, Hazes JM: Measurement of morning stiffness in rheumatoid arthritis clinical trials. J Clin Epidemiol 1997, 50:757-763.

22. Lehman AJ, Esdaile JM, Klinkhoff AV, Grant E, Fitzgerald A, Canvin $\mathrm{J}$ : A 48-week, randomized, double-blind, double-observer, placebo-controlled multicenter trial of combination methotrexate and intramuscular gold therapy in rheumatoid arthritis: results of the METGO study. Arthritis Rheum 2005, 52:1360-1370.

23. Felson DT, Anderson JJ, Boers M, Bombardier C, Furst D, Goldsmith C: American College of Rheumatology preliminary definition of improvement in rheumatoid arthritis. Arthritis Rheum 1995, 38:727-735.

24. Pincus T, Stein C: $A C R-20$ : clinical or statistical significance? Arthritis Rheum 1999, 42:1572-1576.

25. Xi C, Tan L, Sun Y, Liang F, Liu N, Xue H, Luo Y, Yuan F, Sun Y, Xi $Y$ : A novel recombinant peptide containing only two T-cell tolerance epitopes of chicken type II collagen that suppresses collagen-induced arthritis. Mol Immunol 2009, 46:729-737.

26. Tanaka $D$, Kagari T, Doi $H$, Shimozato T: Administration of antitype II collagen antibody sustains footpad swelling of mice caused by a delayed-type hypersensitivity reaction and induces severe arthritis. Clin Exp Immuno/ 2007, 148:360-367.

27. Cazzola M, Antivalle M, Sarzi-Puttini $P$, Dell'Acqua D, Panni B Caruso I: Oral type II collagen in the treatment of rheumatoid arthritis. A six-month double blind placebo-controlled study. Clin Exp Rheumatol 2000, 18:571-577.

28. Myers LK, Higgins GC, Finkel TH, Reed AM: Juvenile arthritis and autoimmunity to type II collagen. Arthritis Rheum 2001, 44:1775-1781.

29. Choy EH, Scott DL, Kingsley GH, Thomas S: Control of rheumatoid arthritis by oral tolerance. Arthritis Rheum 2001, 44:1993-1997.

30. Meyer O: Oral immunomodulation therapy in rheumatoid arthritis. Joint Bone Spine 2000, 67:384-392.

31. Min SY, Hwang SY, Park KS, Lee JS, Lee KE, Kim KW: Induction of IL-10-producing CD4 $+\mathrm{CD}^{2} 5^{+} \mathrm{T}$ cells in animal model of collagen-induced arthritis by oral administration of type II Collagen. Arthritis Res Ther 2004, 6:R213-R219.

32. Tong T, Zhao W, Wu YQ, Chang Y, Wang QT, Zhang LL, Wei W: Chicken type II collagen induced immune balance of Th subtypes in mesenteric lymph node lymphocytes in rats with collagen-induced arthritis. Inflamm Res 2009 in press.

33. Faria $A M$, Weiner $\mathrm{HL}$ : Oral tolerance: therapeutic implications for autoimmune diseases. Clin Dev Immunol 2006, 13:143-157.

34. Bäckström NF, Dahlgren UI: Bystander suppression of collageninduced arthritis in mice fed ovalbumin. Arthritis Res Ther 2004, 6:R151-R160.

35. Oliveira CR, Taniguchi EA, Fusaro AE, Victor JR, Brito CA, Duarte $\mathrm{AJ}$, Sato MN: Bystander effect in synergy to anergy in oral tol- 
erance of Blomia tropicalis/ovalbumin murine co-immunization model. J Clin Immunol 2005, 25:153-161.

36. Staines NA, Derry CJ, Marinova-Mutafchieva L, Ali N, Davies DH, Murphy JJ: Constraints on the efficacy of mucosal tolerance in treatment of human and animal arthritic diseases. Ann NY Acad Sci 2004, 1029:250-259.

37. Corrigall VM, Panayi GS: Autoantigens and immune pathways in rheumatoid arthritis. Crit Rev Immunol 2002, 22:281-293.

38. Sieper J, Kary S, Sörensen H, Alten R, Eggens U, Hüge W, Hiepe $F$, Kühne A, Listing J, Ulbrich N, Braun J, Zink A, Mitchison NA: Oral type II collagen treatment in early rheumatoid arthritis. A double-blind, placebo-controlled, randomized trial. Arthritis Rheum 1996, 39:41-51.

39. Höer A, Gothe H, Schiffhorst G, Sterzel A, Grass U, Häussler B: Comparison of the effects of diclofenac or other non-steroidal anti-inflammatory drugs (NSAIDs) and diclofenac or other NSAIDs in combination with proton pump inhibitors (PPI) on hospitalisation due to peptic ulcer disease. Pharmacoepidemiol Drug Saf 2007, 16:854-858. 